\title{
Diet and Physical Activity in the Prevention of Colorectal Cancer
}

Mamta Mehta, MD, MPH, and Moshe Shike, MD

\begin{abstract}
Diet has been linked to the prevention of colorectal cancer (CRC) and may explain some of the differences in incidence and mortality among various populations. Evidence suggests that a high intake of red and processed meats is associated with an increased risk of CRC. The protective benefits of fiber are unclear, although in some studies fiber is associated with reduced CRC risk. The role of supplements, such as calcium, vitamin D, and folic acid, remains uncertain, and these nutrients cannot be currently recommended for chemoprevention. Obesity and sedentary lifestyle have been associated with an increased risk for colon cancer. Because of the inherent difficulty in studying the effects of specific nutrients, dietary pattern analysis may be a preferable approach to the investigation of the relationship between diet and risk for human diseases. Lifestyle modifications, such as increasing physical activity and consumption of a diet rich in fiber, fruits, vegetables, fish, and poultry and low in red and processed meats, have been advocated for primary prevention of several chronic diseases, and may in fact be beneficial for cancer prevention, particularly CRC. (J Nat/ Compr Canc Netw 2014;12:1721-1726)
\end{abstract}

Colorectal cancer (CRC) is the fourth most common cancer diagnosed in the United States. It is estimated that in 2014 there will be 136,830 new cases of and 50,310 deaths from CRC, second only to lung cancer. ${ }^{1}$ Worldwide, CRC is the second most common cancer diagnosed in women and the third in men.

The genesis of CRC is multifactorial and complex; genetic, familial, dietary, lifestyle, and environmental factors

From Memorial Sloan Kettering Cancer Center, New York, New York.

Submitted March 9, 2014; accepted for publication July 8, 2014. The authors have disclosed that they have no financial interests, arrangements, affiliations, or commercial interests with the manufacturers of any products discussed in this article or their competitors.

Correspondence: Mamta Mehta, MD, MPH, Memorial Sloan Kettering Cancer Center, 1275 York Avenue, New York, NY 10065 E-mail: mehtam@mskcc.org all play important roles. It is clear that in well-defined inherited forms of CRC, such as familial polyposis and Lynch syndrome, the inherited genetic predisposition is decisive and the role of other factors may be limited to nonexistent. However, in most CRC cases-the so called "sporadic" cases - the effect of other factors such as diet may be important. To add to the complexity, it is clear that genetic-environmental interactions may ultimately determine the effects of genetic, nutritional, or environmental factors. Most studies on diet and cancer have analyzed data with little or no consideration of these interactions, which have only recently become evident. Despite these difficulties, current information implicates significant effects of diet on CRC risk. Given that diet is modifiable, it is an area that deserves consideration and investigation.

The purpose of studies on diet and specific diseases is to formulate dietary recommendations. These recommendations should also promote general health and do not consider reduction of CRC risk in isolation. Countries where dietary patterns have become more Westernized have seen a parallel incremental increase in CRC incidence. ${ }^{2,3} \mathrm{Mi}$ gration studies show that immigrants from low-incidence regions who move to high-incidence industrialized regions may incur an elevated lifetime risk for CRC compared with those who remain in the low-incidence regions. ${ }^{4,5}$ Considering that approximately $75 \%$ of patients with CRC have sporadic disease, ${ }^{6}$ these findings highlight the potential impact of dietary and environmental influences on the overall incidence of and mortality from CRC.

Epidemiologic and laboratory animal studies performed during the past 40 years suggest a link between nutritional factors and colorectal carcinogenesis. ${ }^{7,8}$ In 2007, the World Cancer Research Fund (WCRF) and the American Institute for Cancer Research (AICR) published an exhaustive evaluation of the literature on the association between diet and various cancers and suggested that changes in diet could significantly reduce the risk of cancer. $^{9}$ The 
evaluation of food and nutrition in the prevention of CRC, in particular, was updated in 2011. ${ }^{10}$

Research on nutrition, however, is extremely challenging because dietary assessment techniques are imprecise in extracting data on consumption of single foods and nutrients. Traditional nutritional analyses of individual foods and nutrients have been valuable but have conceptual and methodologic limitations.11 People do not eat isolated nutrients. Meals are complex combinations of specific nutrients that are likely to interact synergistically. 12 Changes in the consumption of one food item can trigger significant changes in other items. Given that dietary consumption varies from meal to meal and day to day, dietary consumption is extremely difficult to survey. This article provides a critical review of the literature on the role of individual foods and nutrients and the effect of dietary patterns and physical activity on the primary prevention of CRC.

\section{Red Meat, Processed Meat, and Fat}

In the updated CRC 2011 Report, the WCRF and AICR validate that the evidence for red and processed meats as a cause of CRC is "convincing," and that poultry and fish are better choices for protein. ${ }^{9,10}$

The mechanism explaining how red and processed meats increase CRC risk is not known. It was initially proposed that risk may be related to animal fat content. ${ }^{13}$ However, 2 large randomized control studies, the Women's Health Initiative Dietary Modification Trial and the Polyp Prevention Trial, failed to prove that a low-fat (20\% of total energy intake) dietary pattern reduces the risk of CRC. ${ }^{14,15}$ In a meta-analysis of 6 cohort and case-control studies, the summary relative risk (RR) estimate for animal fat intake and CRC was insignificant (RR, 1.15; 95\% CI, 0.93-1.42). ${ }^{16}$

Several other mechanisms have been proposed to explain the effects of red and processed meats on colon carcinogenesis. Cooking meat at high temperature or on an open flame produces heterocyclic amines (HCAs) and polycyclic aromatic hydrocarbons (PAHs), which have been shown to be potent carcinogens in animal studies. ${ }^{17}$ When using well-done meat as a surrogate marker for HCAs, epidemiologic studies confirm a positive association with CRC. ${ }^{18}$ Additionally, genetic polymorphisms may increase the susceptibility of some individuals to HCAs. ${ }^{19,20}$ Dietary heme iron, which is 10 -fold higher in red meat than in white, may be a pro-carcinogenesis agent. ${ }^{21}$ Heme iron induces DNA damage, catalyzes peroxidation of fat in foods, and induces colon cytotoxicity. ${ }^{22}$ Lastly, nitrites from cured meats react with amines to generate $N$-nitroso compounds, which may be carcinogenic to colonocytes. ${ }^{23}$

In a dose-response meta-analysis of case-control and cohort studies, Norat et $\mathrm{al}^{24}$ concluded that if average red meat consumption decreased to $70 \mathrm{~g}$ per week, risk for CRC would decline by $7 \%$ to $24 \%$ in countries where consumption is high. However, results from epidemiologic studies on meat consumption and CRC risk have been inconsistent because of difficulty in acquiring precise nutritional data, and potential confounders such as lifestyle factors, familial and genetic predisposition, and environmental factors. In a large prospective Japanese cohort study, higher consumption of red meat was associated with an increased risk of colon cancer among women (RR, 1.48; 95\% CI, 1.01-2.17). Higher total meat consumption was associated with colon cancer among men (RR, 1.44; 95\% CI, 1.06-1.98), but not red meat consumption. No risk association between meat consumption and rectal cancer was found..$^{25}$ In the Danish Diet, Cancer, and Health cohort study, no association was found between total meat consumption and CRC, but the risk for CRC was elevated in consumers of lamb (RR, 1.07; 95\% CI, 1.02-1.13). Rectal cancer risk was elevated in those with higher intake of pork (RR, 1.18; 95\% CI, 1.02-1.36). ${ }^{26}$

In summary, evidence suggests a positive association between high consumption of red and processed meats and CRC, although the evidence is inconclusive.

\section{Fiber, Fruits, and Vegetables}

In the 1960s, Burkitt ${ }^{27}$ first proposed that fiber may be protective against CRC. He observed that African Blacks who consumed high-fiber and low-fat foods had a lower incidence of colon cancer and mortality compared with their white counterparts with an intake of low-fiber and high-fat foods. Subsequent epidemiologic and cohort studies looking at the role of fiber in reducing the risk of CRC yield inconsistent results although evidence for the prevention of $\mathrm{CRC}$ was recently upgraded to "convincing" by WCRF and AICR. ${ }^{10}$ This may be partially explained by the fact that fiber is not a specific chemical entity but rather a diverse group of compounds with different physiochemical properties that can affect 
the colon mucosa in different ways. Furthermore, major sources of dietary fiber vary across studies. Cereals are a major contributor to dietary fiber intake described in the European cohort studies, whereas fruits and vegetables are the main sources of fiber reported in North American cohort studies. ${ }^{28}$ Considering fiber content solely misses the point that other vital nutrients may differ depending on the source of fiber.

Dietary fiber, by definition, is not digested by human enzymes. Some fibers, however, may be metabolized by colonic bacteria producing a variety of compounds that may affect not only the colonic mucosa but also the diversity of the bacterial population in the microbiome. Because of the recent recognition that colonic bacteria may exert profound metabolic, genetic, and immunologic effects on the host, ${ }^{29}$ the study of fiber and its effects on colon carcinogenesis, and health in general, may need to be addressed, with a special focus on its effects on the microbiome.

Potential protective mechanisms of fiber include dilution of fecal carcinogens, reduction in transit time of feces through the bowel, binding of carcinogenic bile acids, and production of short-chain fatty acids..$^{30}$ Short-chain fatty acids are the main energy compounds in colonocytes.

In a pooled analysis of 13 prospective cohort studies, fiber intake was associated with a $16 \%$ reduction in CRC risk in the highest quintile group compared with the lowest (RR, 0.84; 95\% CI, 0.77-0.92). However, when adjusting for age and additional nutritional and lifestyle factors, only a nonsignificant weak inverse association was found (RR, 0.94; 95\% CI, 0.86-1.03). ${ }^{27}$ In the NIH-AARP Diet and Health prospective cohort study, fibers from grains, fruits, vegetables, and beans were inversely associated with CRC risk. After adjustment for other risk factors, these inverse relationships essentially disappeared, except for fiber from grains (multivariate RR, 0.86; 95\% CI, 0.76-0.98). ${ }^{31}$

Randomized trials reveal mixed results, particularly on the association between fiber and recurrence of precancerous colon polyps. In the Polyp Prevention Trial, no difference was seen in adenoma recurrence or rectal mucosal proliferation rate over a 4 -year period between subjects in groups randomized to a diet high in fiber $(18 \mathrm{~g} /$ $1000 \mathrm{kcal})$ and low in fat (20\% of total energy intake) diet and controls. ${ }^{17,32}$ However, there was variation in compliance. When examining data from subjects who were most adherent to the diet, there was a $35 \%$ reduction in adenoma recurrence compared with controls. ${ }^{33}$
In another randomized study, subjects given a high wheat bran fiber supplementation (13.5 g/d) compared with a lower amount $(2.0 \mathrm{~g} / \mathrm{d}) \mathrm{did}$ not have protection against recurrent colorectal adenomas after a median of 34 months. ${ }^{34}$

Reports examining the effect of fruit and vegetable consumption on CRC risk in general suggest a weak inverse association. Case-control studies report strong evidence for an inverse association, whereas several reviews and meta-analyses of cohort studies have not found significant inverse associations between fruit and vegetable intake and CRC risk. ${ }^{35,36}$ Recall and selection biases may hamper the interpretation of these case-control studies by overestimating the association, whereas imprecise determination of dietary intake may underestimate the protective effect of fruits and vegetables on CRC risk in the prospective cohort studies. Furthermore, an observed inverse association between fruit and vegetable intake and CRC risk may be weakened when adjusting for potential confounders such as physical activity, obesity, alcohol consumption, and smoking. ${ }^{37,38}$

Because of the serious methodologic difficulties in studying the effects of fiber and the recent recognition that it may have an impact on health and disease through its effect on the microbiome, a rigorous examination is needed. Nevertheless, increasing the fiber consumption from the average intake in the Western diet (12-14 g/d) to a more desirable level $(25-30 \mathrm{~g} / \mathrm{d})$ can be recommended. Such an increase can be achieved by consuming more fruits, vegetables, and whole grains, and may also lower the risk of other chronic conditions, such as heart disease. ${ }^{39}$

\section{Vitamins and Minerals}

Various minerals and micronutrients have been suggested to be protective against CRC. Calcium is thought to protect the colon mucosa by binding to toxic secondary bile acids and forming insoluble complexes, thereby reducing proliferation and stimulating differentiation of colon epithelial cells..$^{40}$ In a pooled analysis of 10 prospective cohort studies, higher consumption of dietary and supplemental calcium was associated with a lower risk of CRC (0.78; 95\% CI, 0.69-0.88). ${ }^{41}$ In individuals with a history of colorectal adenomas, a meta-analysis of 2 randomized trials revealed an $18 \%$ risk reduction in the recurrence of adenomas in patients taking calcium compared with placebo over a 4-year period (RR, 
Mehta and Shike

0.82; 95\% CI, 0.69-0.98).42 Another randomized study revealed that the effect of calcium persisted for a least 5 years after cessation of treatment. ${ }^{43} \mathrm{Al}$ though these results are promising, long-term studies are needed to confirm and quantify the potential chemopreventive effect and safety of calcium in CRC prevention.

Vitamin D has been shown to have antiproliferative, prodifferentiating, and proapoptotic effects in colonocytes, but whether the actions of vitamin D require calcium or are exerted independently is not clear. ${ }^{44,45}$ In a meta-analysis of 9 prospective studies, the pooled RR for CRC was lower for the category of highest vitamin D intake (RR, 0.88; 95\% CI, 0.80-0.96) and blood levels of 25-hydroxy vitamin D (RR, 0.67; 95\% CI, 0.54-0.80) compared with the lowest category. ${ }^{46}$ However, in the Women's Health Initiative randomized clinical trial, women who took a combination of calcium $(1000 \mathrm{mg}$ ) plus vitamin D (400 IU) did not have a lower risk for CRC after a mean of 7 years compared with the placebo group (RR, 1.08; 95\% CI, 0.86-1.34). ${ }^{47}$ Hence, data thus far do not support a recommendation for the general use of calcium plus vitamin D supplementation for preventing CRC.

Folic acid plays an essential role in DNA synthesis. DNA methylation abnormalities during colorectal carcinogenesis may be affected by a lack of adequate folic acid. In a mouse model, microarray gene expression analysis demonstrated the role of folic acid supplementation in altering certain gene expressions involved in colon carcinogenesis and preventing adenoma formation. ${ }^{48}$ In a randomized controlled study of 860 Chinese men older than 50 years with no endoscopic findings of adenomas, participants who took $1 \mathrm{mg} / \mathrm{d}$ of folic acid, particularly those with a low baseline folic acid level, had a lower rate of adenoma on repeat colonoscopy 3 years later (RR, 0.49; 95\% CI, 0.37-0.63) compared with participants given placebo. ${ }^{49}$ This result suggests that folic acid may exert a protective effect on normal colon mucosa. However, these findings cannot be generalized, because they may apply only to populations with a high prevalence of folic acid deficiency. However, epidemiologic studies have found that folic acid deficiency is highly prevalent in some developing countries where the incidence of CRC is relatively low.

Most prospective studies report an inverse association between folic acid intake and CRC risk. In a pooled analyses of 13 studies, high total folic acid intake (dietary and supplemental sources) was associated with reduced CRC risk (RR, 0.85; 95\% CI, $0.77-0.95) .{ }^{50}$ With mandatory implementation of folic acid fortification in the United States in 1998, there was concern that excess folic acid might, in fact, increase CRC risk through enhancing DNA synthesis in proliferating cells. ${ }^{51}$ Stevens et a ${ }^{152}$ examined total folic acid intake (dietary and supplemental) and CRC risk in the postfortification period over 8 years (1999-2007) in a prospective cohort study and found that, in fact, high total folic acid intake was still associated with a lower risk of CRC (RR, 0.81; 95\% CI, 0.66-0.99).

Other dietary micronutrients, such as vitamins $\mathrm{A}, \mathrm{C}$, and $\mathrm{E}$, are thought to have anticarcinogenic effects based on their antioxidant properties suggested by observational studies. ${ }^{53-55}$ However, these studies did not examine specific vitamins, but deduced the level of their intake from the diet. Thus, any protective effect may have resulted from other dietary factors present in the vitamin-containing foods. Randomized trials have not supported a role for the use of antioxidants for protection against CRC..$^{56,57}$

\section{Dietary Patterns}

The impact of macronutrients and micronutrients on CRC prevention is thus far inconclusive. Recognizing that nutrients may act synergistically, nutrition epidemiologists have examined whether dietary patterns or combinations of foods, rather than specific nutrients, are associated with CRC risk.

Dixon et $\mathrm{a}^{58}$ observed 2 predominant dietary patterns in 3 large European prospective cohorts: the Veg pattern, characterized by intake of vegetables, legumes, fruits, pasta, poultry, and fish; and the PPP pattern, characterized by increased intake of pork, processed meats, potatoes, and saturated fats. In Swedish women, a PPP diet conferred an increased risk of colon cancer (RR, 1.62; 95\% CI, 1.12-2.34), whereas in Finnish men, an increased risk for rectal cancer was seen (RR, 2.21; 95\% CI, 1.07-4.57). In a Japanese prospective cohort, Kim et $\mathrm{a}^{159}$ found that the Western dietary pattern (characterized by meat, poultry, cheese, bread, and butter) was positively associated with colon cancer risk in women (RR, 2.21; 95\% CI, 1.10-4.45). Because the evidence for the general health benefits of the Mediterranean diet, Bamia et $\mathrm{al}^{60}$ investigated its relationship 
to $\mathrm{CRC}$ risk in the European Prospective Investigation into Cancer and Nutrition (EPIC) cohort and found an $8 \%$ to $11 \%$ decrease in CRC risk in subjects most adherent to the Mediterranean diet compared with those who were least adherent. Most recently, evidence has suggested that eating a diet high in sugar, saturated fats, and other foods that promote inflammation can drive the growth of many cancers, including CRC. ${ }^{61}$ Consuming fewer proinflammatory nutrients, such as red and processed meats and refined sugary drinks, and more anti-inflammatory plant-based nutrients and whole grains may reduce the risk of gastrointestinal tract cancer death.

\section{Obesity and Physical Activity}

More than one-third of US adults (35.7\%) are estimated to be obese. ${ }^{62}$ The association between body weight and different cancers is now well recognized. For colon cancer, the RR attributable to obesity has been estimated to be 1.24 for men (95\% CI, 1.21-1.28) and 1.09 (95\% CI, 1.05-1.14) for women, but may vary with country of origin. ${ }^{63}$ Various anthropometric variables, such as body mass index, waist circumference, and waist/hip ratio, are suggested to be positively associated with colon cancer in both men and women. ${ }^{64}$ Common metabolic pathways linking obesity and CRC risk have been hypothesized, such as those of chronic inflammation, oxidative stress, and insulin resistance.

Physical activity may protect against colon cancer through decreasing inflammation, increasing the body's metabolic efficiency and capacity, and reducing insulin resistance. ${ }^{10}$ In a meta-analysis of 16 cohort studies, individuals who were the most physically active were observed to have a $23 \%$ to $24 \%$ lower risk of colon cancer than those who were least active. For rectal cancer, no statistically significant association was observed. ${ }^{65}$

\section{Conclusions}

An abundance of data exists in the literature on the role of nutrition, obesity, and physical activity in modulating CRC risk. Dietary regimens characterized by a high consumption of fiber, fruits, vegetables, fish, and poultry, and lower in red and processed meats seem to exert protective effects against CRC and are also good for the general health. Evidence suggests a positive link between obesity and CRC risk, although whether weight loss can lead to CRC risk reduction is unclear. Physical activity has been associated with reduced CRC risk but also plays an important role in improving cardiovascular health.

Research in nutrition continues to be a challenge, because dietary consumption varies across countries and cultures. In the experimental setting, it is difficult to change people's diets and maintain consistency. Furthermore, the effect of dietary changes on carcinogenesis may be subtle and may not be appreciated in a typical 3- to 5-year study. Therefore, long-term studies are needed to survey the impact of dietary patterns on CRC prevention. Comparing the regional differences between these dietary patterns may also be insightful.

\section{References}

1. SEER Stat Fact Sheets: Colon and Rectum Cancer. Surveillance, Epidemiology, and End Results Program Web site. Available at: http://seer. cancer.gov/statfacts/html/colorect.html. Accessed June 8, 2014.

2. Zhang $B$, Li $X, N a k a m a ~ H$, et al. A case-control study on risk of changing food consumption for colorectal cancer. Cancer Invest 2002;20:458-463.

3. Bejar LM, Gili M, Infantes B, Marcott PF. Effects of changes in dietary habits on colorectal cancer incidence in twenty countries from four continents during the period 1971-2002. Rev Esp Enferm Dig 2011;103:519-529.

4. Whittermore AS, Wu-Williams AH, Lee M, et al. Diet, physical activity and colorectal cancer among Chinese in North America and China. J Natl Cancer Inst 1990:82:915-926.

5. Monroe KR, Hankin JH, Pike MC, et al. Correlation of dietary intake and colorectal cancer incidence among Mexican-American migrants: the multiethnic cohort study. Nutr Cancer 2003;45:133-147.

6. Genetics of Colorectal Cancer. National Cancer Institute at the National Institute of Health Web site. Available at: http://www.cancer. gov/cancertopics/pdq/genetics/colorectal/HealthProfessional. Accessed November 12, 2013.

7. Spiller GA, Freeman HJ. Recent advances in dietary fiber and colorectal diseases. Am J Clin Nutr 1981;34:1145-1152.

8. Burkitt DP. Epidemiology of cancer of the colon and rectum. Cancer 1971;28:3-13.

9. World Cancer Research Fund/American Institute for Cancer Research. Food, Nutrition, Physical Activity, and the Prevention of Cancer: A Global Perspective. Available at: http://www.dietandcancerreport.org/cancer_ resource_center/downloads/Second_Expert_Report_full.pdf. Accessed November 3, 2014.

10. World Cancer Research Fund/American Institute for Cancer Research. Colorectal Cancer 2011 Report: Food, Nutrition, Physical Activity, and the Prevention of Colorectal Cancer. Available at: http://www. dietandcancerreport.org/cancer_resource_center/downloads/cu/ColorectalCancer-2011-Report.pdf. Accessed November 3, 2014.

11. Hu F. Dietary pattern analysis: a new direction in nutritional epidemiology. Curr Opin Lipidol 2002;13:3-9.

12. Jacobs DR, Steffen LM. Nutrients, foods, and dietary patterns as exposures in research: a framework for food synergy. Am J Clin Nutr 2003;78(Suppl):508S-513S.

13. Willett WC, Stampfer MJ, Colditz GA, et al. Relation of meat, fat, and fiber intake to the risk of colon cancer in a prospective study among women. $\mathrm{N}$ Engl J Med 1990;323:1664-1672.

14. Beresford $\mathrm{SA}$, Johnson $\mathrm{KC}$, Ritenbaugh $\mathrm{C}$, et al. Low-fat dietary pattern and risk of colorectal cancer: The Women's Health Initiative Randomized Controlled Dietary Modification Trial. JAMA 2006;295:643-654.

15. Schatzkin A, Lanza E, Corle D, et al. Lack of effect of a low-fat, high-fiber diet on the recurrence of colorectal adenomas. N Engl J Med 2000;342:11491155 .

16. Alexander DD, Cushing CA, Lowe KA, Sceurman B, Roberts MA. Metaanalysis of animal fat or animal protein intake and colorectal cancer. Am J Clin Nutr 2009;89:1402-1409. 
17. Sugimura T, Wakabayashi $K$, Nakagama H, Nagao M. Heterocyclic amines: mutagens/carcinogens produced during cooking of meat and fish. Cancer Sci 2004;95:290-299.

18. Zheng W, Lee SA. Well-done meat intake, heterocyclic amine exposure, and cancer risk. Nutr Cancer 2004;61:437-446.

19. Zhenming F, Shrubsole M, Li G, et al. Using gene-environment interaction analyses to clarify the role of well-done meat and heterocyclic amine exposure in the etiology of colorectal polyps. Am J Clin Nutr 2012;96:1119-1128.

20. Wang $H$, Yamamoto JF, Caberto $C$, et al. Genetic variation in the bioactivation pathway for polycyclic hydrocarbons and heterocyclic amines in relation to risk for colorectal neoplasia. Carcinogenesis 2010;32:203-209.

21. Pierre F, Tache S, Petit CR, et al. Meat and cancer: haemoglobin and haemin in a low-calcium diet promote colorectal carcinogenesis at the aberrant crypt stage in rats. Carcinogenesis 2003;24:1683-1690.

22. Sesink AL, Termont DS, Kleibeuker JH, Van der meer R. Red meat and colon cancer: the cytotoxic and hyperproliferative effects of dietary heme. Cancer Res 1999;59:5704-5709.

23. Santarelli RL, Vendeuvre JL, Naud N, et al. Meat processing and colon carcinogenesis: cooked, nitrite-treated, and oxidized high-heme cured meat promotes mucin-depleted foci in rats. Cancer Prev Res (Phila) 2010;3:852864.

24. Norat T, Lukanova A, Ferrari P, Riboli E. Meat consumption and colorectal cancer risk: Dose-response meta-analysis of epidemiological studies. Int J Cancer 2002;98:241-256.

25. Takachi R, Tsubono $\mathrm{Y}, \mathrm{Baba} \mathrm{K}$, et al. Red meat intake may increase the risk of colon cancer in Japanese, a population with relatively low red meat consumption. Asia Pac J Clin Nutr 2011;20:603-612.

26. Egeberg R, Olsen A, Christensen J, et al. Associations between red meat and risks for colon and rectal cancer depend on the type of red meat consumed. J Nutr 2013;143:464-472.

27. Burkitt DP. Related disease-related cause? Lancet 1969;2:1229-1231.

28. Park Y, Hunter DJ, Spiegelman D, et al. Dietary fiber intake and risk of colorectal cancer: a pooled analysis of prospective cohort studies. JAMA 2005;294:2894-2857.

29. Abreu MT, Peek RM Jr. Gastrointestinal malignancy and the microbiome. Gastroenterology 2014;146:1534-1546.

30. Bingham SA. Mechanisms and experimental and epidemiological evidence relating dietary fibre (non-starch polysaccharides) and starch to protection against large bowel cancer. Proc Nutr Soc 1990;49:153-171.

31. Schatzkin A, Mouw T, Park Y, et al. Dietary fiber and whole-grain consumption in relation to colorectal cancer in the NIH-AARP Diet and Health Study. Am J Clin Nutr 2007;85:1353-1360.

32. Pfeiffer R, McShane L, Wargovich M, et al. The effect of a low-fat, high fiber, fruit and vegetable intervention on rectal mucosal proliferation. Cancer 2003;98:1161-1168.

33. Sansbury L, Wanke K, Albert PS, et al. The effect of strict adherence to a high-fiber, high-fruit and -vegetable, and low-fat eating pattern on adenoma recurrence. Am J Epidemiol 2009;170:576-584.

34. Alberts DS, Martinez ME, Roe DJ, et al. Lack of effect of a high-fiber cereal supplement on the recurrence of colorectal adenomas. Phoenix Colon Cancer Prevention Physicians' Network. N Engl J Med 2000;342:11561162.

35. Riboli E, Norat T. Epidemiologic evidence of the protective effect of fruit and vegetables on cancer risk. Am J Clin Nutr 2003;78(Suppl):559S-569S.

36. Koushik A, Hunter DJ, Spiegelman D, et al. Fruits, vegetables, and colon cancer risk in a pooled analysis of 14 cohort studies. J Natl Cancer Inst 2007;99:1471-1483.

37. Aune D, Lau R, Chan DS, et al. Nonlinear reduction in risk for colorectal cancer by fruit and vegetable intake based on meta-analysis of prospective studies. Gastroenterology 2011;141:106-118.

38. Vogtmann E, Xiang Y, Li H, et al. Fruit and vegetable intake and the risk of colorectal cancer: results from the Shanghai Men's Health Study. Cancer Causes Control 2013;24:1935-1945.

39. Ning H, Van Horn L, Shay CM, Lloyd-Jones DM. Associations of dietary fiber intake with long-term predicted cardiovascular disease risk and C-reactive protein levels (from the National Health and Nutrition Examination Survey Data [2005-2010]). Am J Cardiol 2014;113:287-291.

40. Newmark HL, Wargovich MJ, Bruce WR. Colon cancer and dietary fat, phosphate and calcium: a hypothesis. J Natl Cancer Inst 1984;72:1323-1325.

41. Cho E, Smith-Warner S, Spiegelman D, et al. dairy foods, calcium, and colorectal cancer: a pooled analysis of 10 cohort studies. J Natl Cancer Inst 2004;96:1015-1022.
42. Carroll C, Cooper K, Papaioannou D, et al. Supplemental calcium in the chemoprevention of colorectal cancer: a systematic review and metaanalysis. Clinical Therapeutics 2010;32:789-803.

43. Grau MV, Baron JA, Sandler RS, et al. Prolonged effect of calcium supplementation on risk of colorectal adenomas in randomized trial. J Natl Cancer Inst 2007;99:129-136.

44. Gonzalez-Sancho JM, Larriba MJ, Oronez-Moran P, et al. Effects of 1alpha,25-dihydroxyvitamin D3 in human colon cancer cells. Anticancer Res 2006;26:2669-2681.

45. Fedriko V, Bostick RM, Flanders WD, et al. Effects of vitamin D and calcium on proliferation and differentiation in normal colon: a randomized clinical trial. Cancer Epidemiol Biomarkers Prev 2009;18:2933-2941.

46. Ma Y, Zhang $P$, Wang F, et al. Association between vitamin $D$ and risk of colorectal cancer: a systematic review of prospective studies. J Clin Oncol 2011;29:3775-3782

47. Wactawski-Wende J, Kotchen JM, Anderson GL, et al. Calcium plus vitamin D supplementation and risk of colorectal cancer. N Engl J Med 2006;354:684-696.

48. Lin YW, Wang JL, Chen HM, et al. Folic acid supplementary reduces the incidence of adenocarcinoma in a mouse model of colorectal cancer: microarray gene expression profile. J Exp Clin Cancer Res 2011;30:116.

49. Gao Q, Chen $H$, Chen $Y$, Wang $Y$ et al. Folic acid prevents the initial occurrence of sporadic colorectal adenoma in Chinese older than 50 years of age: a randomized clinical trial. Cancer Prev Res (Phila) 2013;6:744-752.

50. Kim DH, Smith-Warner SA, Spiegelman D, et al. Pooled analyses of 13 prospective cohort studies on folate intake and colon cancer. Cancer Causes Control 2010;21:1919-1930.

51. Mason JB, Dickstein A, Jacques PF, et al. A temporal association between folic acid fortification and an increase in colorectal cancer rates may be illuminating important biological principles: a hypothesis. Cancer Epidemiol Biomarkers Prev 2007;16:1325-1329.

52. Stevens VL, McCullough ML, Sun J, et al. High levels of folate from supplements and fortification are not associated with increased risk of colorectal cancer. Gastroenterology 2011;141:98-105.

53. La Veccchia, Braga C, Negri E, et al. Intake of selected micronutrients and risk of colorectal cancer. Int J Cancer 1997;73:525-530.

54. Bostick RM, Potter JD, McKenzie DR, et al. Reduced risk of colon cance with high intake of vitamin E: the Iowa Women's Health Study. Cancer Res 1993;53:4230-4237.

55. Kune G, Watson L. Colorectal cancer protective effects and the dietary micronutrients folate, methionine, vitamins B6, B12, C, E, selenium and lycopene. Nutr Cancer. 2006;56:11-21.

56. Papaioannou D, Cooper KL, Carroll C, et al. Antioxidants in the chemoprevention of colorectal cancer and colorectal adenomas in the general population: a systematic review and meta-analysis. Colorectal Dis 2011;13:1085-1099.

57. Bjelakovic G, Nikolova D, Simnetti RG, Gluud C. Antioxidant supplements for prevention of gastrointestinal cancers: a systematic review and metaanalysis. Lancet 2004;364:1219-1228.

58. Dixon LB, Balder HF, Virtanen MJ, et al. Dietary patterns associated with colon and rectal cancer: results from the Dietary Patterns and Cancer (DIETSCAN) Project. Am J Clin Nutr 2004;80:1003-1011.

59. Kim MK, Sasaki S, Otani T, Tsugane S. Dietary patterns and subsequent colorectal cancer risk by subsite: a prospective cohort study. Int J Cancer 2005;115:790-798.

60. Bamia C, Lagiou P, Buckland G, et al. Mediterranean diet and colorectal cancer risk: results from a European cohort. Eur J Epidemiol 2013;28:317328.

61. Tabung FK, Steck SE, Zhang J, et al. Dietary inflammatory index and risk of mortality: findings from the Aerobics Center Longitudinal Study. Presented at the American Institute for Cancer Research (AICR) Annual Research Conference; November 7, 2013; Bethesda, MD.

62. Ogden CL, Carroll MD, Kit BK, Flegal KM. Prevalence of obesity in the United States, 2009-2010. NCHS Data Brief 2012;(82):1-8.

63. Reneham AG, Tyson M, Egger M, et al. Body-mass index and incidence of cancer: a systematic review and meta-analysis of prospective observational studies. Lancet 2008;371:569-578.

64. Keimling M, Renehan AG, Behrens G, et al. Comparison of associations of body mass index, abdominal adiposity, and risk of colorectal cancer in a large prospective cohort study. Cancer Epidemiol Biomarkers Prev 2013;22:13831394.

65. Robsahm TE, Aagnes B, Hjartaker A, et al. Body mass index, physical activity, and colorectal cancer by anatomical subsites: a systematic review and meta-analysis of cohort studies. Eur J Cancer Prev 2013;22:492-505. 